\title{
Preface
}

\section{Limb Alignment: The Key to Success}

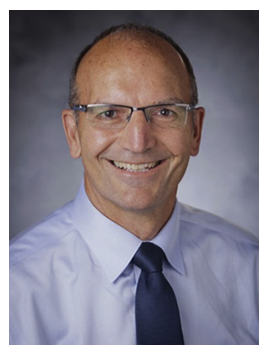

Annunziato (Ned) Amendola, MD

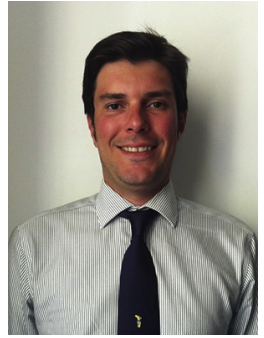

Davide Edoardo Bonasia, MD

Editors

In the young active patient with early knee osteoarthritis, chondral defects, meniscal deficiency, and/or ligament instability, it is crucial to assess knee alignment. In this issue of Clinics in Sports Medicine, you will appreciate the importance of limb alignment and how it is central to biologic reconstruction around the knee. It is important to have an armamentarium of techniques for the varus and valgus knees, meniscal transplantation combined with osteotomy, anterior cruciate ligament instability, and osteotomy. Each article is designed to be concise, give all the pertinent details, and really give the reader an opportunity to learn all aspects of the procedure. Although osteotomies have been around for a long time, the indications and techniques have improved, yielding more predictable results. In addition to the standard techniques currently utilized, we have included an article on navigation (Parker and colleagues) and accuracy of limb realignment. In many situations, especially with osteoarthritis, the disease process is already quite advanced. We all realize the outcome of osteotomy is dependent on the condition of the knee joint and yields poorer results in advanced osteoarthritis. Therefore, consideration and assessment for joint overload and earlier diagnosis and indications before advanced changes may be the way we should be looking at things, as in the article by Giffin and colleagues, "Degenerative meniscal tears and high tibial osteotomy." Do current treatment algorithms need to be realigned?" This special issue of Clinics in Sports Medicine has assembled experts from around the world to draw an overview of the use of osteotomy, patient evaluation, 
xvi Preface

techniques, indications, and results, aiming to provide up-to-date information on a hot topic that seems to be always in continuous development.

Annunziato (Ned) Amendola, MD Urbaniak Sports Sciences Institute

Duke University

3475 Erwin Road

Durham, NC 27705, USA

Davide Edoardo Bonasia, MD Department of Orthopaedics and Traumatology AO Ordine Mauriziano "Umberto I" Hospital University of Torino Via LAmarmora 26 10128, Torino, Italy

E-mail addresses: ned.amendola@duke.edu (A. Amendola) davidebonasia@virgilio.it (D.E. Bonasia) 